G. Grdenić, M. Delimar and J. Beerten, "Assessment of AC network modeling impact on smallsignal stability of AC systems with VSC HVDC converters," International Journal of Electrical Power \& Energy Systems, vol. 119, Jul. 2020.

Digital Objective Identifier: https://doi.org/10.1016/j.ijepes.2020.105897

URL:

https://www.sciencedirect.com/science/article/pii/S0142061519336154

NOTICE: this is the author's version of a work that was accepted for publication in the International Journal of Electrical Power \& Energy Systems. Changes resulting from the publishing process, such as peer review, editing, corrections, structural formatting, and other quality control mechanisms may not be reflected in this document. Changes may have been made to this work since it was submitted for publication. A definitive version was subsequently published in the International Journal of Electrical Power \& Energy Systems, vol. 119, Jul. 2020.

(C) 2020.This manuscript version is made available under the CC-BY-NC-ND 4.0

license http://creativecommons.org/licenses/by-nc-nd/4.0/ 


\title{
Assessment of AC Network Modeling Impact on Small-Signal Stability of AC Systems with VSC HVDC Converters
}

\author{
G. Grdenić (1)* ${ }^{*}$ M. Delimar ${ }^{(1)}$, J. Beerten ${ }^{(2),(3)}$ \\ ${ }^{(1)}$ Faculty of Electrical Engineering and Computing, University of Zagreb, Unska 310000 Zagreb, Croatia \\ ${ }^{(2)}$ Department of Electrical Engineering, KU Leuven, Kasteelpark Arenberg 10 postbus 2440, 3001 Leuven, Belgium \\ ${ }^{(3)}$ Energyville, Thor Park 8310, 3600 Genk, Belgium
}

\begin{abstract}
The increasing number of flexible ac transmission systems (FACTS) and high voltage direct current (HVDC) systems is changing perspectives on small-signal stability in ac grids, as traditional steady-state modeling does not allow representing higher-frequency interactions between synchronous generators, ac grid and power electronic-based devices. Since dynamically modeling all grid components typically result in an overly complex system model with a very high order, hybrid network models have been put forward as a compromise to increase the scope of the study, whilst keeping the overall system model order manageable. They do so by combining grid dynamics in the vicinity of the power electronic devices with steady-state assumptions for the rest of the network. This paper examines the impact of converter and grid parameters on the outcome of the stability analysis through comparative examples of a double multi-infeed (MI) HVDC system and a single and double HVDC infeed in the IEEE 39 bus system and by using either steadystate or dynamic line models. Doing so, it determines the most relevant parameters to the boundary selection between a dynamic and a static ac grid model in hybrid network models.
\end{abstract}

Keywords: hybrid network model, small-signal stability, steady-state network model, voltage source converter HVDC

${ }^{*}$ The corresponding author 


\section{Introduction}

In power system studies, small-signal stability analysis (SSSA) has traditionally been used to identify low-damped electromechanical oscillations between synchronous generators (SG) [1]. Since these oscillations occur at low frequencies, it has been commonplace to model the ac network by means of algebraic or steady-state equations for the analysis, thereby neglecting faster electromagnetic transients. With more and more power electronics-based equipment entering the transmission system, the frequency range over which dynamic interactions occur is increasing, and consequently the conventional view on small-signal stability in power system is changing accordingly. In order to be able to identify and mitigate problems w.r.t. these faster interactions between synchronous generators, ac grid and power electronic-based devices, SSSA should also be able to capture these phenomena. In this sense, the steady-state ac network model no longer suffices and dynamic line models are needed.

Researchers in [2] showed that dynamic based ac line modeling is necessary for accurately assessing the system's small signal stability for the case of two line commutated converter (LCC) high voltage direct current (HVDC) lines in a multi-infeed (MI) HVDC system. Moreover, the comparative analysis in [3] on a point-to-point voltage source converter (VSC) link suggests that steady-state modeling of the Thevenin ac network equivalent cannot always reflect the actual stability of the system, even when mainly focusing on slower system modes. Reflecting on the observations from [2] and [3], the steady-state network modeling can give rise to misleading conclusions about the system stability margin. As a result, dynamic modeling of the ac network has found its way into a number of recent small-signal studies. Usually, however, these are small ac systems, like island systems [4] or systems where the external ac network is represented with Thevenin equivalents, as has been done, for example, in $[5,6,7,8]$. Some researchers have started 
using dynamic modeling of the ac network also in larger grids, as has been carried out in [9, 10, 11].

Dynamic modeling of the entire ac network, however, implies a significant increase in the number of state variables of the system model, and consequently of the size of the state-space matrix of the linearized system, necessary for determining the system's eigenvalues. As long as the size of the ac grid is small this does not produce difficulties, but in large multi-machine ac networks, dynamic modeling of all static elements (transmission lines, power transformers, capacitors, and inductors) can lead to very large state-space matrices from which it can become difficult to calculate the eigenvalues [12]. Moreover, it is not clear whether dynamically representing all elements is strictly necessary for an accurate assessment of small-signal stability of the system.

One solution to this problem is to model the ac network dynamically only in the vicinity of power electronic-based devices and keep the rest of the network modeled using algebraic steadystate equations. This approach has been proposed in [13], resulting in a hybrid network model for small-signal stability analysis of power systems. However, the boundary selection between these two approaches, i.e. static and dynamic ac network modeling has not been examined in more detail. Similar modeling partition can also be found in some other work which is not exclusively related to SSSA. For example, in [14], a platform for analyzing power systems has been developed in which the entire power system is modeled as a multivariable feedback control system (FCS) and divided similarly into dynamic and static parts. The developed FCS model has been verified on the IEEE 9 bus test system with HVDC links (both LCC and VSC), though, the selection of the boundary between static and dynamic parts has not been discussed in the paper. However, defining the boundary between dynamically and statically modeled sections of an ac grid is not an unambigous problem with a straightforward solution which holds in all possible cases. The primary 
goal of this research is therefore to systematically investigate the impact of relevant converter and grid parameters to this boudary selection. First, the influence that ac line modeling has on system small-signal stability properties is demonstrated on the generic example of a VSC-based MI-HVDC system. Afterward, converter and grid parameters have been assessed in order to identify the most relevant ones to the boundary selection between statically and dynamically modeled parts of an ac grid. This has been carried out by the comparison procedure based on small-signal stability analysis in the case of a single and double VSC HVDC infeed in a relatively larger ac network.

The rest of the article is organized as follows. Section II describes the assembling of steadystate, dynamic and hybrid network models and establishes the comparison procedure of different system models. Section III introduces the case of a generic VSC-based MI-HVDC system and in Section IV the assessment of converter and grid parameters has been carried out in the IEEE 39 bus grid with VSC HVDC converters. The main findings are summarized in the conclusion section.

\section{Methodology}

\subsection{Modeling}

The studied systems include three types of network elements: voltage source converters, synchronous generators and ac lines. For the modeling of the voltage source converter, an ideal lossless average modular multilevel converter with simplified internal dynamics is used. It is adjusted from [15] and described by the $20^{\text {th }}$ order system with its parameters taken from [16]. Generally, the model can be described by a set of differential and algebraic equations:

$$
\begin{aligned}
& \dot{\mathbf{x}}_{\mathrm{vsc}}=\mathbf{f}\left(\mathbf{x}_{\mathrm{vsc}}, \mathbf{u}_{\mathrm{vsc}}, \mathrm{v}_{\mathrm{vsc}}\right) \\
& \mathrm{i}_{\mathrm{vsc}}=\mathbf{g}\left(\mathbf{x}_{\mathrm{vsc}}, \mathbf{u}_{\mathrm{vsc}}, \mathrm{v}_{\mathrm{vsc}}\right)
\end{aligned}
$$


where $\mathbf{x}_{\mathrm{vsc}}$ and $\mathbf{u}_{\mathrm{vsc}}$ are the converter state and input vectors, $\mathrm{v}_{\mathrm{vsc}}$ is the voltage at the point of common coupling and $i_{v s c}$ is the corresponding injection current expressed in the $d$-q reference frame.

For the synchronous generator modeling, a $6^{\text {th }}$ order model [17] is used together with the AC4A excitation system model [18]. It can be also represented succinctly with the following set of differential and algebraic equations:

$$
\begin{aligned}
& \dot{\mathbf{x}}_{\text {gen }}=\mathbf{f}\left(\mathbf{x}_{\text {gen }}, \mathbf{u}_{\text {gen }}, \dot{i}_{\text {gen }}\right) \\
& v_{\text {gen }}=\mathbf{g}\left(\mathbf{x}_{\text {gen }}, \mathbf{u}_{\text {gen }}, \dot{i}_{\text {gen }}\right)
\end{aligned}
$$

where $\mathbf{x}_{\text {gen }}$ and $\mathbf{u}_{\text {gen }}$ are the generator state and input vectors, $v_{\text {gen }}$ is the voltage at the connection point and $i_{\text {gen }}$ is the corresponding injection current expressed in the q-d reference frame.

The ac network is modeled in a global D-Q reference system, synchronous generators in their local q-d reference systems and converters in their local d-q reference systems. Corresponding transformations have been applied in order to integrate the individual elements into an ac/dc system. Depending on how the ac network is modeled, different overall system models have been established.

\subsubsection{Steady-state network model}

In this system model, the dynamics of the ac grid, i.e. ac lines and transformers, are neglected. The ac grid is represented by the set of algebraic (nodal) equations using a standard admittance matrix $\mathbf{Y}_{\text {bus }}$ assembled with network parameters evaluated at the nominal frequency:

$$
\mathbf{i}_{\text {bus }}=\mathbf{Y}_{\text {bus }} \times \mathbf{v}_{\text {bus }}
$$

where $\mathbf{i}_{\text {bus }}$ and $\mathbf{v}_{\text {bus }}$ are the vectors of injection currents and bus voltages, respectively. The converter and generator models described by (1) and (2) are combined with the admittance matrix 
as described in [1]. Loads are modeled as constant admittances and included in the admittance matrix.

\subsubsection{Dynamic network model}

In the dynamic network model, the PI line model has been employed. The adequacy of the PI line model has been validated for the observed line lengths and for the frequencies within the bandwidth of interest (the converter's control). The dynamics of the line currents $\mathrm{i}_{\mathrm{LINE}}=i_{\text {LINE,D }}+$ $j \cdot i_{L I N E, Q}$ and bus voltages $\mathrm{v}=v_{D}+j \cdot v_{Q}$ in this system model are taken into account and described with the following differential equations in the global D-Q reference frame:

$$
\begin{gathered}
\frac{d}{d t} \mathrm{i}_{\mathrm{LINE}}=\frac{\omega_{b}}{l_{a}} \mathrm{v}_{\mathrm{S}}-\frac{\omega_{b}}{l_{a}} \mathrm{v}_{\mathrm{R}}-\left(\frac{\omega_{b} \cdot r_{a}}{l_{a}}+j \cdot \omega_{b} \cdot \omega_{g}\right) \cdot \mathrm{i}_{\mathrm{LINE}} \\
\frac{d}{d t} \mathrm{v}=\frac{\omega_{b}}{c_{a}} \sum \mathrm{i}_{\mathrm{LINE}}-j \cdot \omega_{b} \cdot \omega_{g} \cdot \mathrm{v}
\end{gathered}
$$

where $v_{S}$ and $v_{R}$ are the sending-end and receiving-end voltages on the transmission line, $\omega_{b}$ and $\omega_{g}$ the base and per-unit grid frequencies, and $l_{a}, r_{a}$ and $c_{a}$ the line inductance, resistance and capacitance, respectively. Doing so, the number of state variables in the system grows significantly since each of the line currents and bus voltages are described with two state variables. The synchronous generator model used in this system model, also includes two additional state variables, describing the stator winding dynamics. Kirchhoff's laws are used for the integration of the grid's currents and voltages with the differential algebraic equations of SG and VSC models.

\subsubsection{Hybrid network model}

In the hybrid network model, the methodology from [13] is adopted and includes a combination of the two previously described approaches. In the vicinity of a power electronic-based device (in this case, a voltage source converter), the ac grid is modeled dynamically, and the rest of the grid is modeled by using the admittance matrix. This way, a number of state variables in a system does 
not grow significantly; and conversely, an accurate system behavior in the vicinity of a powerelectronic device is preserved. The voltage source converter model is combined with the dynamic grid model by using Kirchhoff's laws establishing the current injection model in the form:

$$
\begin{aligned}
& \dot{x}_{\text {dyn }}=f\left(x_{\text {dyn }}, u_{d y n}, v_{d y n}\right) \\
& i_{d y n}=g\left(x_{d y n}, u_{d y n}, v_{d y n}\right)
\end{aligned}
$$

where $\mathbf{x}_{\mathbf{d y n}}$ and $\mathbf{u}_{\mathbf{d y n}}$ are the state and input vectors of the dynamic network area, and $\mathbf{v}_{\mathbf{d y n}}$ and $\mathbf{i}_{\mathbf{d y n}}$ are the vectors of voltages and injection currents at the boundary between dynamic and steadystate network area. Subsequently, this dynamic network area model is integrated with the admittance matrix of the rest of the grid which is modeled in a steady-state.

\subsection{Comparison procedure}

For the comparison of different system models, a quantitative measure based on eigenvalue and participation factor analysis is established. Different system models are built and linearized using Matlab/Simulink. The size of the state-space matrix, and consequently the number of eigenvalues, is the highest for a dynamic model and the lowest for a steady-state model, with a hybrid model being situated in-between. Therefore, for comparison purposes, only the eigenvalues for which the normalized participation of state variables of a power electronic-based device (voltage source converter) has the dominant share, are used:

$$
\lambda_{i} \in A_{X} \mid \sum_{x_{k} \in B} p\left(x_{k}, \lambda_{i}\right)>0.5
$$

where $A_{X}$ is the set of eigenvalues $\lambda_{i}$, related to the voltage source converter obtained from the system model $X ; B$ is the set of state variables $x_{k}$ describing the VSC model.

The thus obtained sets of eigenvalues of the different system models are compared by calculating the total sum error of the damping of the eigenvalues related to the same state variables: 


$$
\varepsilon_{\zeta}=\sum_{\substack{\lambda_{i} \in A_{X 1} \\ \lambda_{j} \in A_{X 2}}} \frac{\operatorname{Abs}\left(\zeta\left(\lambda_{j}\right)-\zeta\left(\lambda_{i}\right)\right)}{\zeta\left(\lambda_{i}\right)}
$$

where $\lambda_{i}$ and $\lambda_{j}$ are the eigenvalues from the sets $A_{X 1}$ and $A_{X 2}$ related to the same state variables and with $\zeta\left(\lambda_{i}\right)$ the mode damping:

$$
\zeta\left(\lambda_{i}\right)=\frac{-\operatorname{Re}\left(\lambda_{i}\right)}{\operatorname{Abs}\left(\lambda_{i}\right)}
$$

The obtained oscillatory modes (related to the voltage source converters) have the frequency of oscillation low enough to be adequately represented by a single PI line model or they are confined to the converter's dc side. Therefore, in the comparison of different system models, the dynamic model of the entire system, is taken as the reference model. The proposed quantitative indicator, that is the total sum error, bundles all eigenvalues related to the VSC model into one single piece of information. Hence, the purpose of this indicator is not a discrimination between highly and poorly damped eigenvalues, but an overall indication of a first comparison of different system models.

\section{Case study 1: multi-infeed system with two converters}

The layout of a generic VSC HVDC multi-infeed system consisting of two converters, representing a reduced ac/dc system model, is shown in Fig. 1. The short-circuit ratio (SCR) at the point of common coupling (PCC) of converter 2 is lower in comparison to the converter $1 \mathrm{SCR}$ since converter 2 is connected to an external grid through an ac line. The state-space representations of two different models of the described system are established: a dynamic model - in which all elements are modeled dynamically and a hybrid model - in which the dynamics of the ac line is neglected. The overall number of state variables in the two system models and by different system elements, are summarized in Table 1. The ac bus voltages are considered to be part of the ac grid 
subsystem state variables. Both converters operate as rectifiers at their nominal power and without reactive power injection into the ac grid.

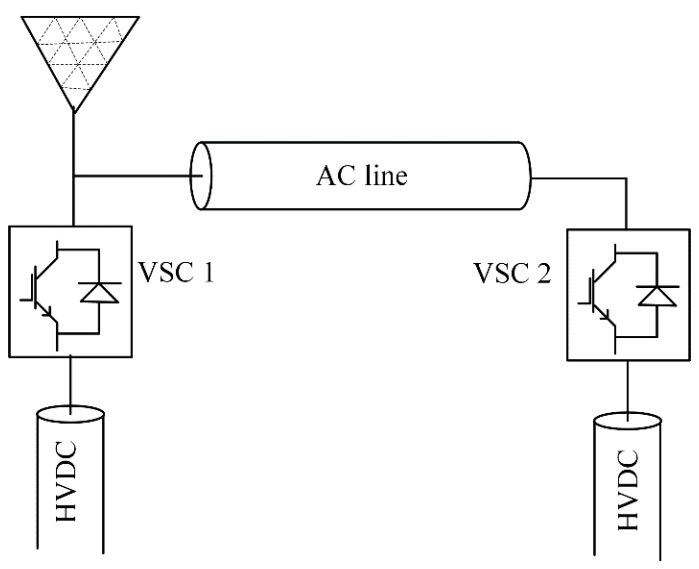

Table 1 Number of state variables in different MI-HVDC models

\begin{tabular}{|c|c|c|}
\hline $\begin{array}{c}\text { Model } \\
\text { elements }\end{array}$ & $\begin{array}{c}\text { Dynamic } \\
\text { model }\end{array}$ & $\begin{array}{c}\text { Hybrid } \\
\text { model }\end{array}$ \\
\hline Converter 1 & 20 & 20 \\
\hline Converter 2 & 20 & 20 \\
\hline Ac line & 6 & 0 \\
\hline $\begin{array}{c}\text { External } \\
\text { grid }\end{array}$ & 2 & 2 \\
\hline Overall & $\mathbf{4 8}$ & $\mathbf{4 2}$ \\
\hline
\end{tabular}

Fig. 1. VSC HVDC multi-infeed layout

Fig. 2 shows the eigenvalues of the system extracted from the state-space matrices of the MIHVDC dynamic and hybrid models and a $150 \mathrm{~km}$ long ac line. System modes slower than 2000 $\mathrm{rad} / \mathrm{s}$ and with real parts larger than -4000 are presented. The green lines represent a $90 \%$ damping boundary; and modes with higher damping are not taken into consideration. A satisfactory matching of the two models can be observed, except for the two modes denoted by Mode 1 and Mode 2. Participation factor analysis of the dynamic model is employed to further analyze these two modes (Figs. 3a and 3b). Mode 1 mostly depends on participation from the state variables related to the d-component of the filtered PCC voltage of converter $2\left(v_{d, f, 2}\right)$ employed as a feedforward term in the inner current control and the d-component of the ac line current $\left(i_{\text {LINE,D }}\right)$. Mode 2 is mostly related to the active power measurement of converter $2\left(p_{a c, m, 2}\right)$, the qcomponent of the filtered PCC voltage of converter $2\left(v_{q, f, 2}\right)$, employed as a feedforward term in the inner current control and the q-component of the ac line current state variable $\left(i_{L I N E, Q}\right)$. The significant participation of the ac line state variables in the observed modes causes a difference between models, since these variables are not present in the hybrid model. Figs. $4 a-4 d$ show the 
converter 1 and converter 2 active and reactive power time-domain responses following a converter 2 active power reference step for four different system models, namely a dynamic nonlinear, dynamic small-signal, hybrid nonlinear and hybrid small-signal model. The nonlinear system models include an ideal lossless time-averaged modular multilevel converter model described by Eq. (1). First, the adequacy of the small-signal models in accurately representing the system dynamics is demonstrated. Second, the difference between the dynamic and the hybrid model can be observed in converter 1 and converter 2 active power, confirming the above statements from the frequency domain.
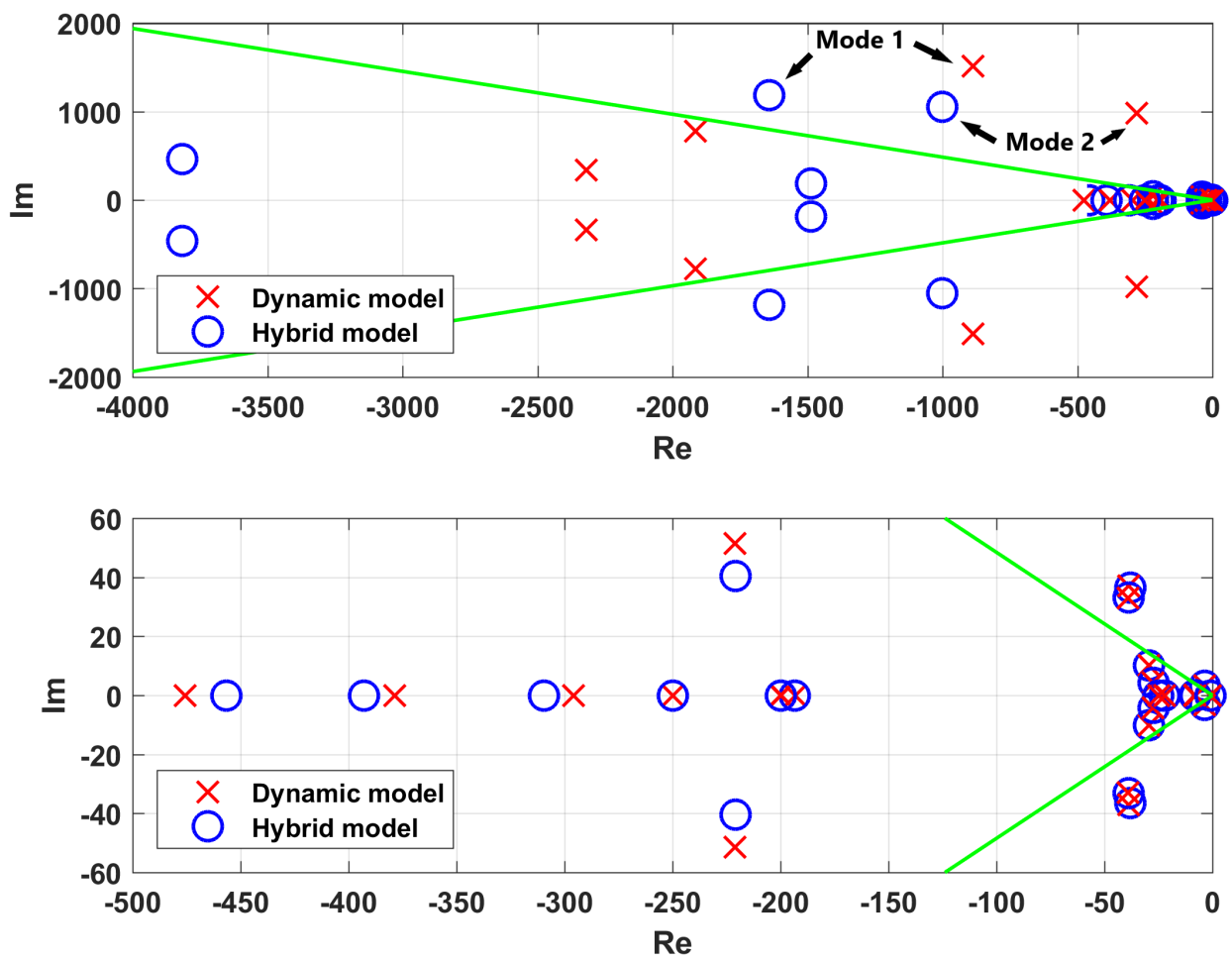

Fig. 2. Eigenvalues of dynamic and hybrid MI-HVDC models 

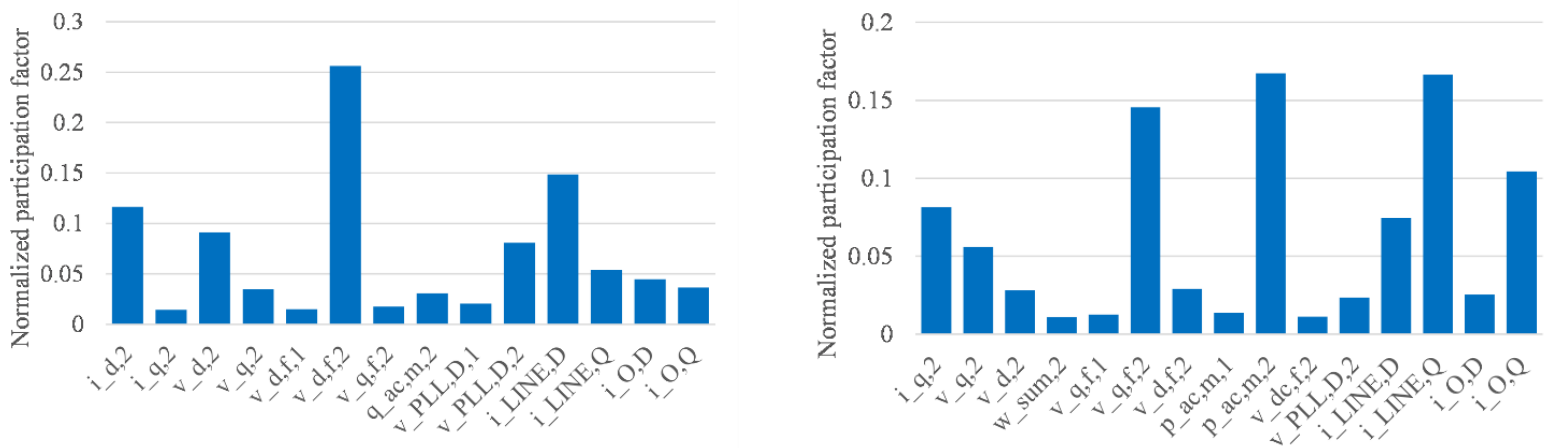

Fig. 3. Normalized participation factors in dynamic model of: a) Mode 1; b) Mode 2

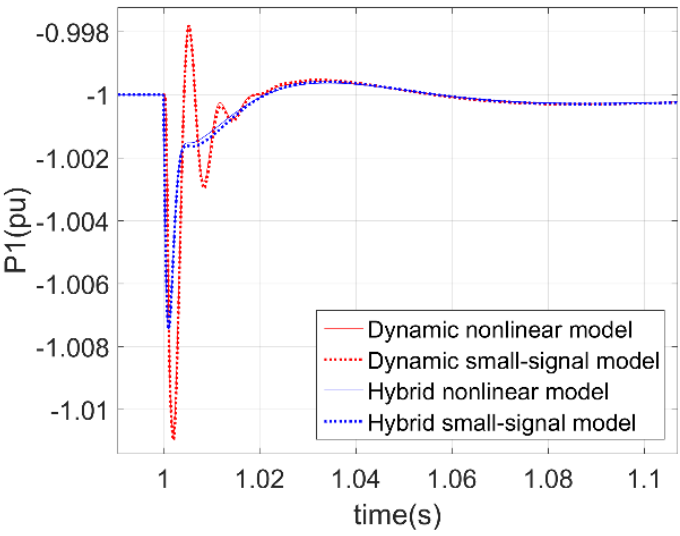

a)

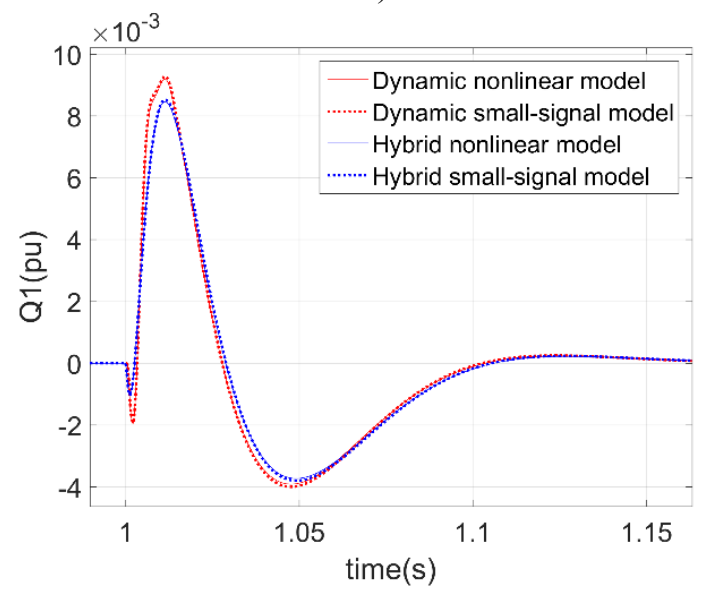

c)

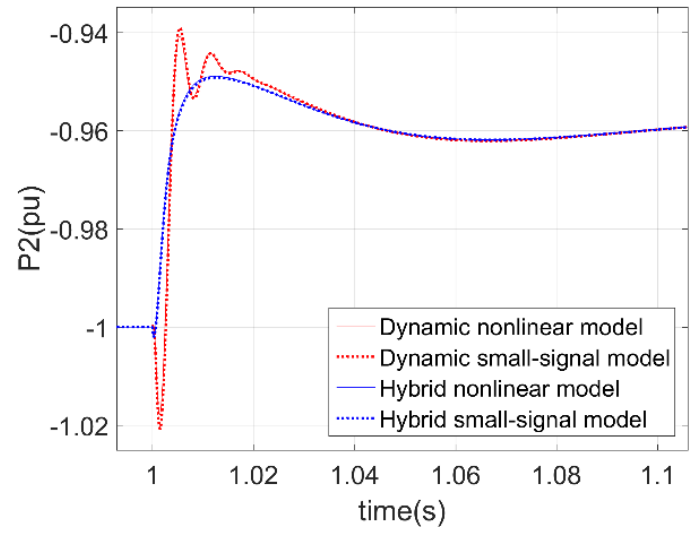

b)

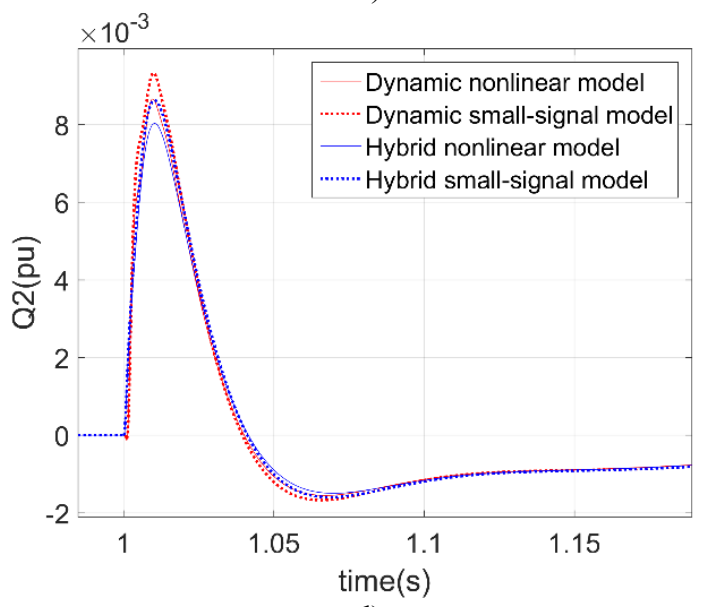

d)

Fig. 4. Time-domain response following an active power reference step at converter 2: a) converter 1 active power; b) converter 2 active power; c) converter 1 reactive power; d) converter 2 reactive power

The influence of the ac line length on damping of the observed modes is demonstrated in Fig.

5a. In the dynamic model, the observed modes become less damped, whereas in the hybrid model 
they remain almost unaltered. Therefore, the difference between the models is increased for longer ac lines. However, in some situations, these modes may become unstable. This is shown in Fig. 5b, demonstrating the influence of the droop coefficient value in the case when the ac voltage droop control is implemented in converter 2 . In the ac voltage droop control, the reactive power reference $q_{a c}^{*}$ is influenced by an ac voltage droop function described with the following expression:

$$
q_{a c}^{*}=k_{d r p}\left(v_{a c}^{r e f}-v_{a c, f}\right)+q_{a c}^{r e f},
$$

where $q_{a c}^{r e f}$ is the external reactive power reference, $v_{a c}^{r e f}$ the ac voltage reference, $v_{a c, f}$ the filtered ac voltage and $k_{d r p}$ the droop coefficient. For values of the coefficient larger than 3.5 , the dynamic model becomes unstable (damping of Mode 2 becomes negative) and this instability is not detected in the hybrid model. The conducted analysis shows an inadequacy of the steady-state ac line model in accurately representing system's small-signal stability.
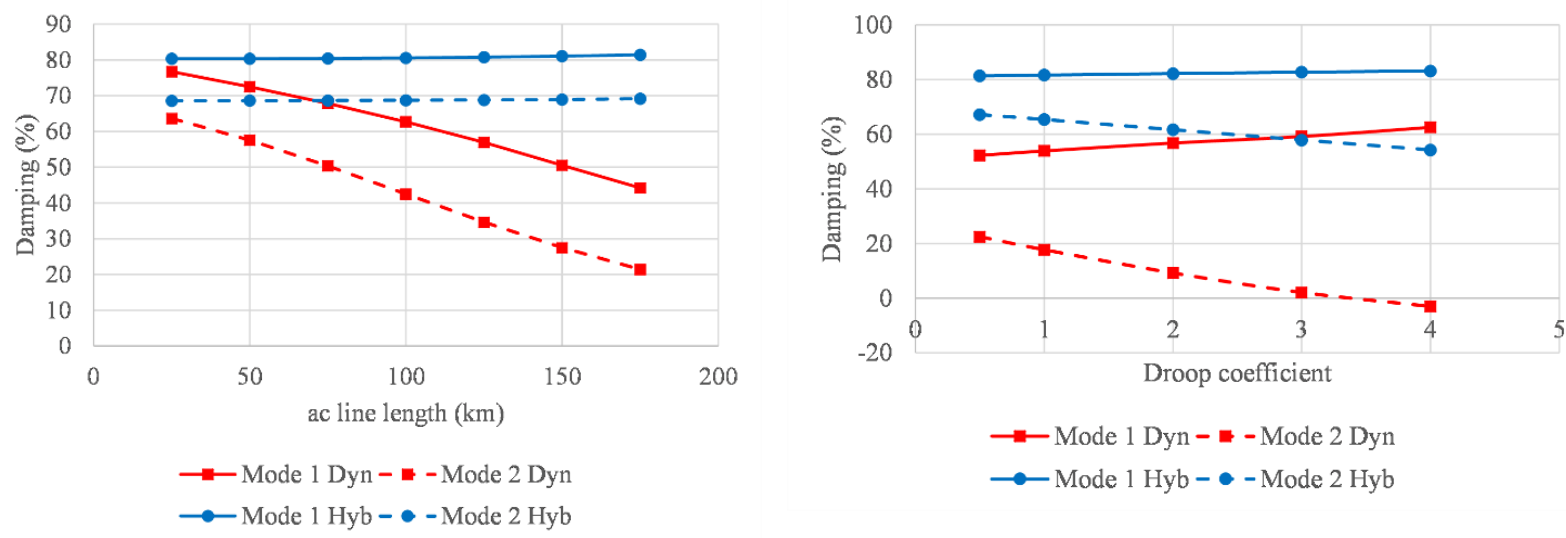

Fig. 5. Mode 1 and Mode 2 damping ratios for different: a) ac line lengths; b) ac voltage droop coefficients

\section{Case study 2: IEEE 39 bus system}

The procedure for comparison of different system models described in section 2.2 is applied to the IEEE 39 bus test system. The parameters of the test system are taken from [19]. First, analyses 
are performed with a single VSC HVDC station in the test system, and afterward with two VSC stations to represent the case with a MI-HVDC system embedded in the grid.

\subsection{Single VSC HVDC station}

First, the influence that location of the VSC HVDC station has on the accuracy of the steadystate model in relation to the dynamic-based model is assessed. In Fig. 6, the total sum error of the steady-state model for the first twelve buses, i.e. when the VSC station is located in each of the buses, is depicted together with the corresponding three-phase SC power at those buses. A clear relationship can be observed: with decreasing SC power, the total sum error of the steady-state model increases. Short-circuit powers are calculated using PSS/E and pre-fault bus voltages from power flow. In continuation, the VSC station installed at the bus with a relatively high SC power - bus 3 - is considered.

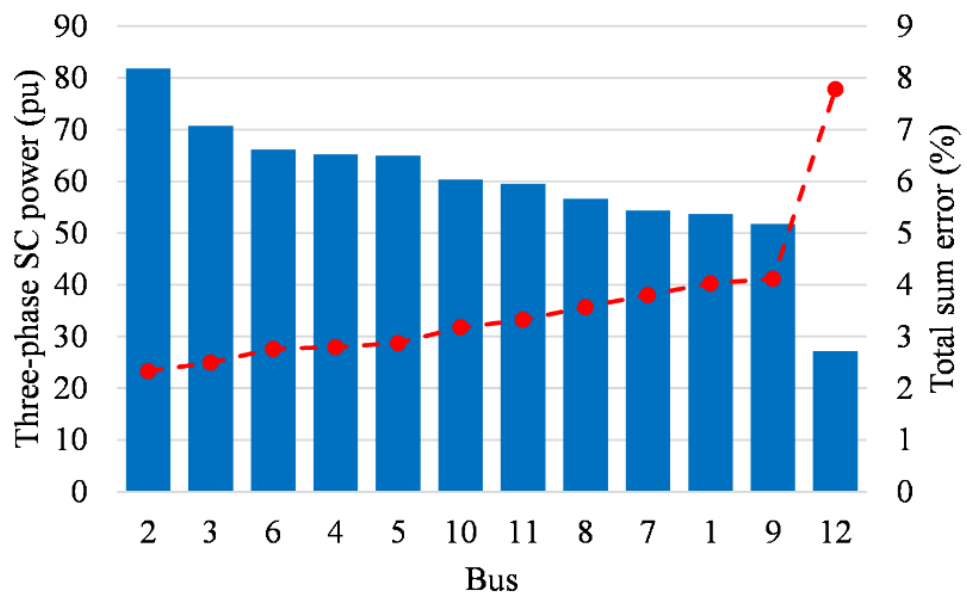

Fig. 6. Total sum error of steady-state model and different locations of VSC station in the grid

Apart from dynamic and steady-state models, two more hybrid models are assembled. In hybrid model 1, bus 3 (connection of VSC) and adjacent lines (Lines 2-3, 3-4 and 3-18) are modeled dynamically, as designated by the red color in Fig. 7. In hybrid model 2, more buses and lines are modeled dynamically - those adjacent to the previously mentioned ones and designated in Fig. 7 
with the blue color. The generator connected to bus 30 , in this case, is also modeled dynamically by taking into account stator dynamics. The number of state variables in different models and in different system elements are summarized in Table 2 . The overall number of state variables in the dynamic model is approximately three times higher in comparison to the steady-state model. The difference between the thus assembled system models is demonstrated in the time-domain for a selected set of system variables, following a converter active power reference step: PLL angle in Figs. $8 \mathrm{a} 1$ and $8 \mathrm{a} 2$, PCC voltage in Figs. $8 \mathrm{~b} 1$ and 8b2, and converter zero-sequence energy-sum in Figs. $8 \mathrm{c} 1$ and $8 \mathrm{c} 2$. First, it can be observed that with more buses and lines modeled dynamically, the response of the system is closer to the response of the full dynamic model (hybrid model 2 in comparison to the steady-state and hybrid model 1). Second, dynamic modeling of the converter's adjacent buses and lines is necessary to precisely capture the dynamics of the system, as can be observed from Fig. $8 b 2$.

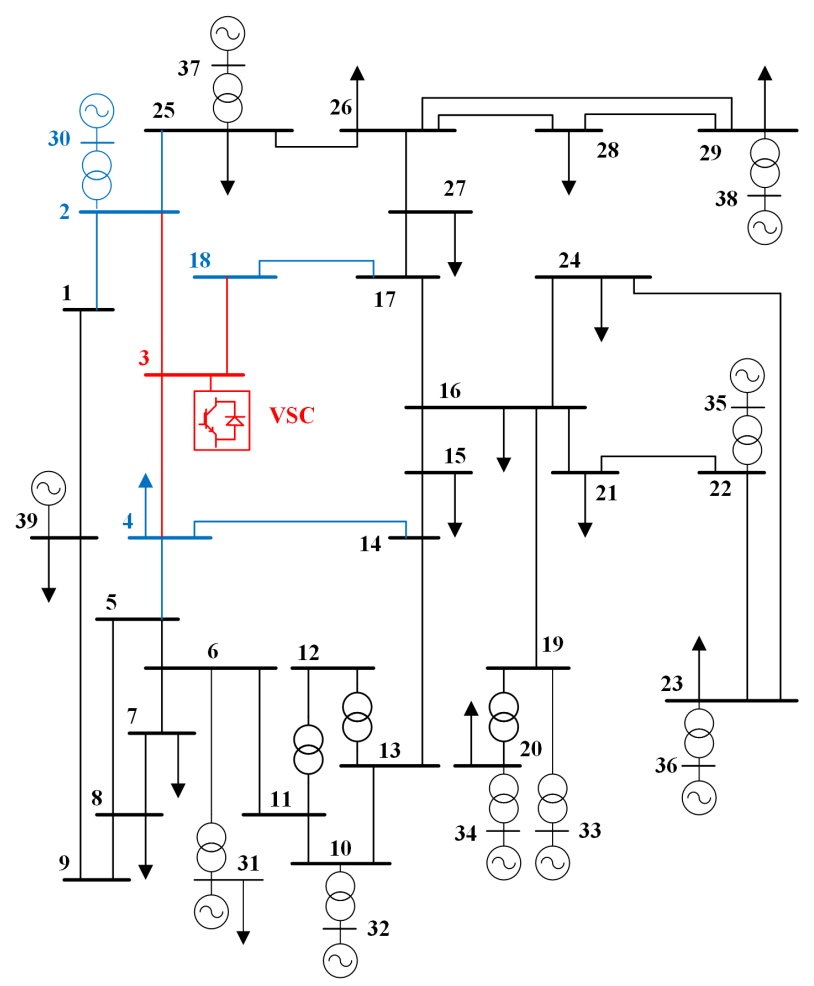

Fig. 7. IEEE 39 bus test system with one VSC station 


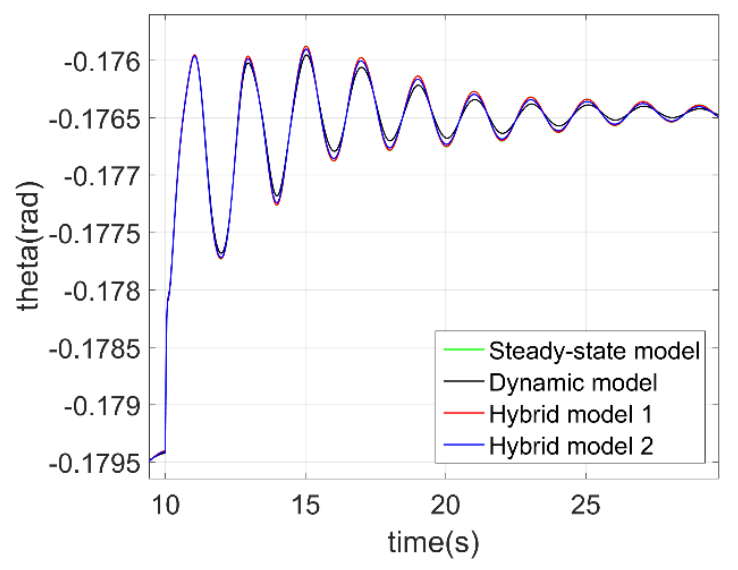

a1)

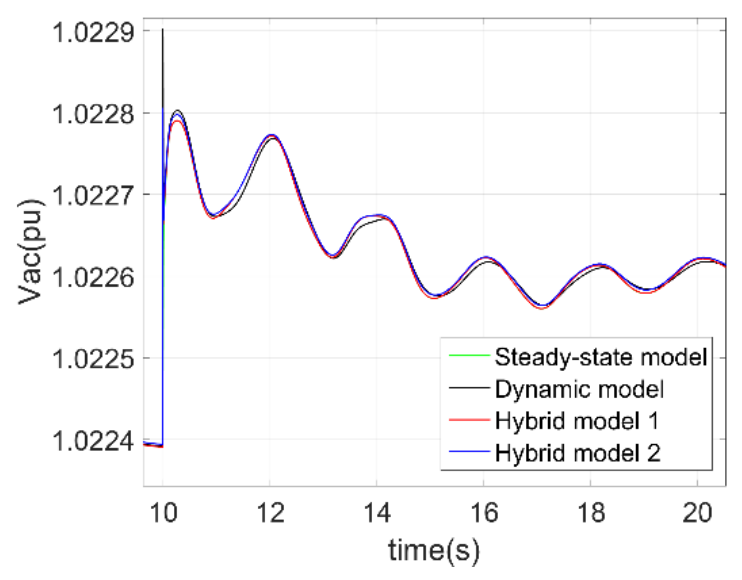

b1)

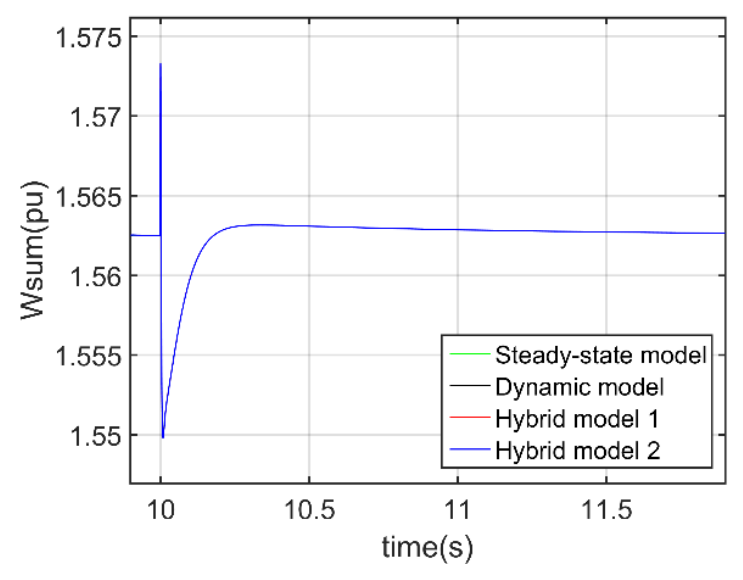

c1)

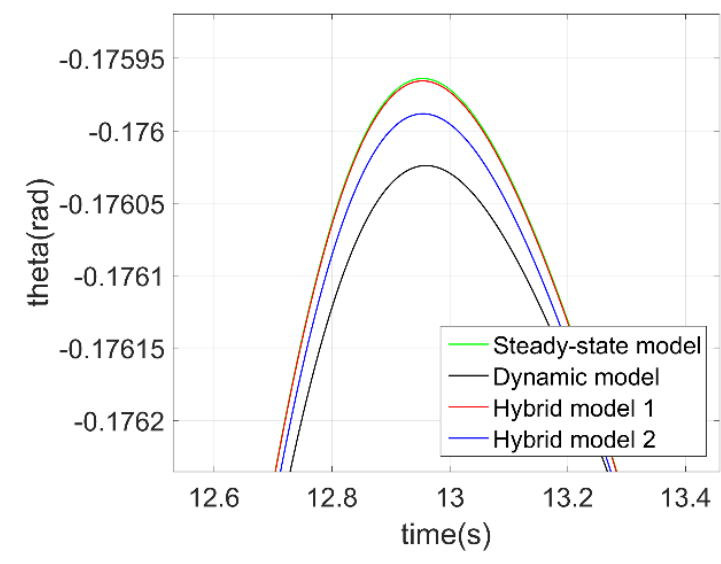

a2)

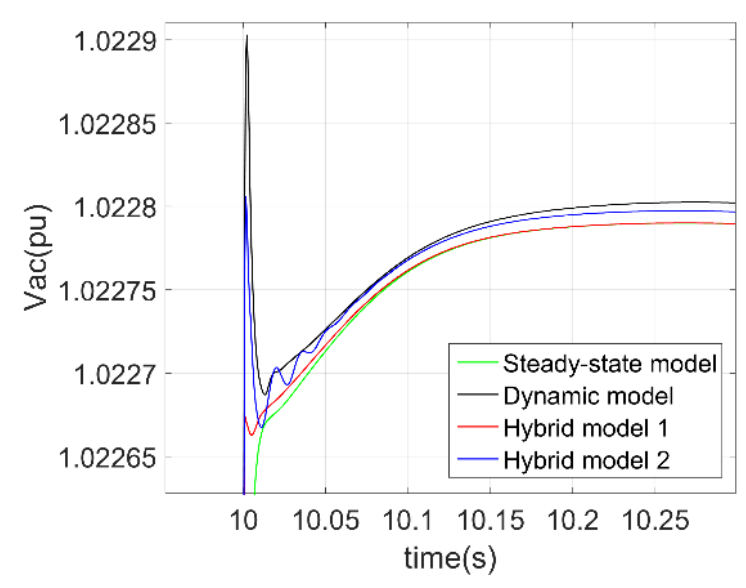

b2)

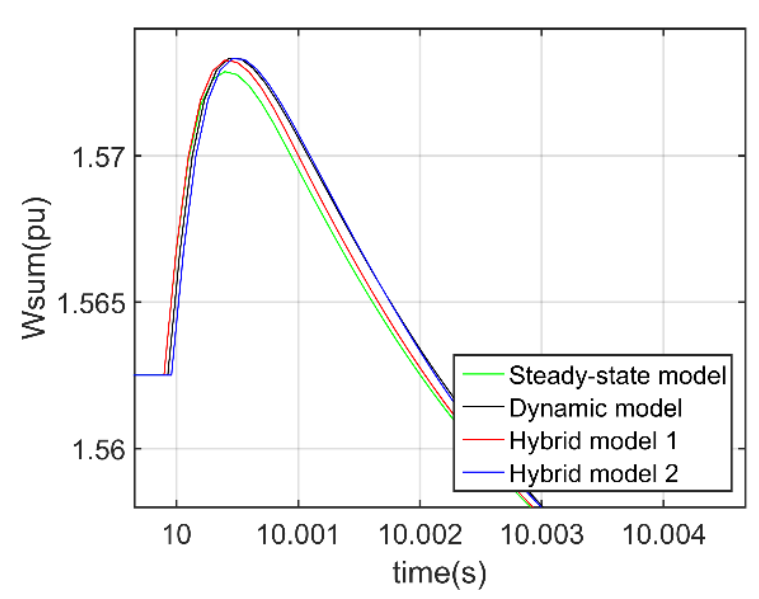

c2)

Fig. 8. Time-domain response following a converter active power reference step: a1) PLL angle; a2) PLL angle (zoom-in); b1) PCC voltage; b2) PCC voltage (zoom-in); c1) Zerosequence energy sum; c2) Zero-sequence energy sum (zoom-in) 
Table 2 Number of state variables in different IEEE 39 models with embedded single VSC station

\begin{tabular}{|c|c|c|c|c|}
\hline $\begin{array}{c}\text { Model } \\
\text { elements }\end{array}$ & $\begin{array}{c}\text { Dynamic } \\
\text { model }\end{array}$ & $\begin{array}{c}\text { Steady-state } \\
\text { model }\end{array}$ & $\begin{array}{c}\text { Hybrid model } \\
\mathbf{1}\end{array}$ & $\begin{array}{c}\text { Hybrid model } \\
\mathbf{2}\end{array}$ \\
\hline Converter & 20 & 20 & 20 & 20 \\
\hline Ac grid & 168 & 0 & 8 & 28 \\
\hline Generators & 90 & 72 & 72 & 74 \\
\hline Overall & $\mathbf{2 7 8}$ & $\mathbf{9 2}$ & $\mathbf{1 0 0}$ & $\mathbf{1 2 2}$ \\
\hline
\end{tabular}

The influence of the following converter parameters on the total sum error of different models has been examined: a phase locked loop (PLL) speed response, an active power control speed response, an active power setpoint, and a type of reactive power control. The speed response of control loops is in close correlation with coefficient values of PI regulators. The parameters of the base case are given in Table 3 and the parameters of different observed cases in Table 4. Case 1 considers slower PLL control, Case 2 slower active power control, Case 3 lower converter active power and Case 4 ac voltage control instead of reactive power control. The results are presented in Fig. 9. It can be observed that the total sum error for each investigated case decreases when more buses and lines are modeled dynamically (hybrid models in comparison to steady-state model). The PLL speed response has the largest influence on the total sum error and the difference between steady-state and hybrid models is the lowest (Case 1). After this follows the type of reactive power control, then the active power setpoint. The speed response of the active power control has the smallest influence on the total sum error amongst the investigated cases. These results are also confirmed by the fact that the relative error of the eigenvalues related to the PLL states are the highest in the base case.

Table 3 Parameters of the base case

\begin{tabular}{|c|}
\hline Phase locked loop $k_{p, P L L}=50 \mathrm{rad} / \mathrm{s}, k_{i, P L L}=833 \mathrm{rad} / \mathrm{s}^{2}$ \\
\hline Active power control $k_{p, p a c}=1 \mathrm{pu}, k_{i, p a c}=50 \mathrm{pu}$ \\
\hline Active power setpoint $P=500 \mathrm{MW}$ \\
\hline Reactive power control $Q=0 \mathrm{Mvar}$ \\
\hline
\end{tabular}


Table 4 Parameters of different observed cases

\begin{tabular}{|c|c|}
\hline Cases & Parameters \\
\hline Case 1 & $k_{p, P L L}=25 \mathrm{rad} / \mathrm{s}, k_{i, P L L}=208 \mathrm{rad} / \mathrm{s}^{2}$ \\
\hline Case 2 & $k_{p, p a c}=0.5 \mathrm{pu}, k_{i, p a c}=25 \mathrm{pu}$ \\
\hline Case 3 & $P=250 \mathrm{MW}$ \\
\hline Case 4 & Ac voltage control $(Q=0 \mathrm{Mvar})$ \\
\hline
\end{tabular}

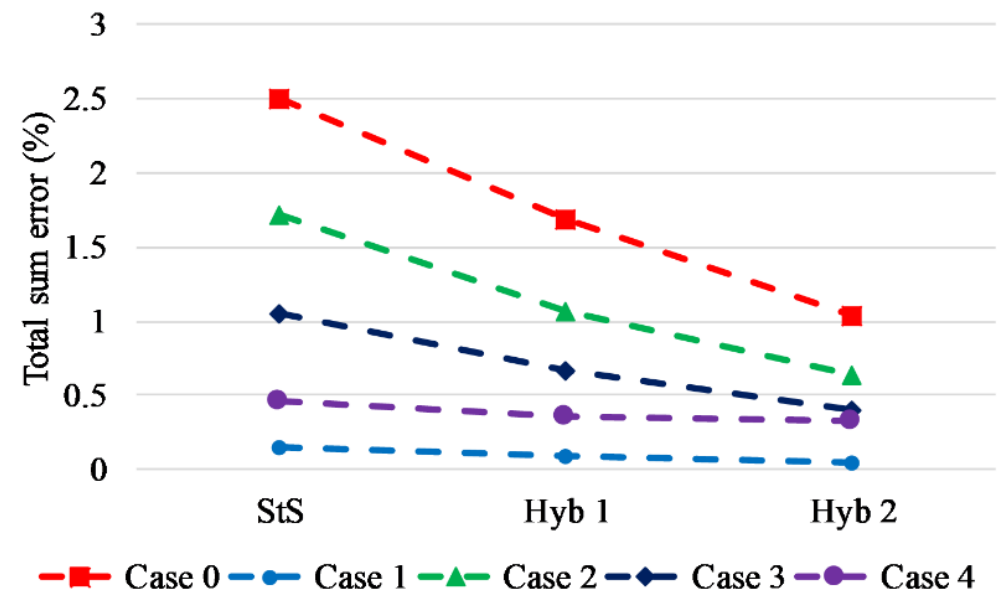

Fig. 9. Total sum error for different observed cases

Since the PLL speed response are found to have the highest impact on the total sum error, the influence of the PLL regulator coefficients are further examined; and results are presented in Fig. 10. The integral gain of the PI regulator is changed proportionally with proportional gain, hence maintaining the same integrator time constant. Apart from the total sum error, Fig. 10 also shows the PLL eigenvalue relative error for different system models labeled with a dotted line. For the values of the PLL proportional gains between 30 and 40, a notable decline in the total sum error and PLL error can be observed. For values outside this range, changes in the total sum error and PLL error are less pronounced. 

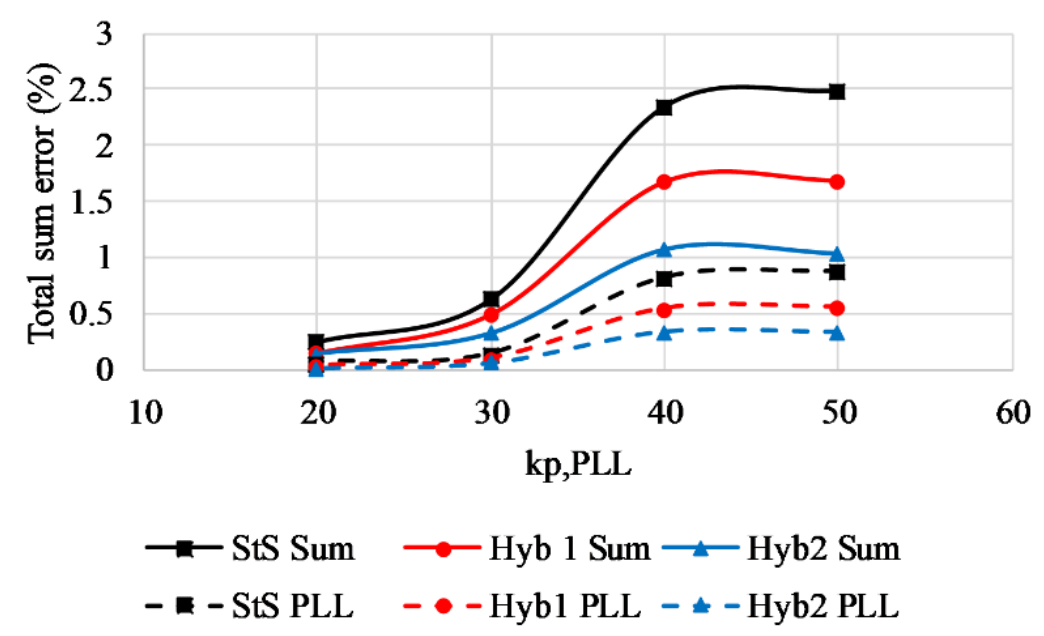

Fig. 10. Total sum error for different PLL proportional gains

\subsection{MI-HVDC system}

Adjacent to the voltage source converter from the previous section, another converter operating as rectifier $(\mathrm{P}=250 \mathrm{MW})$ is connected to neighboring bus 4 (Fig. 11). In this case, three levels of hybrid models have been assessed with the following buses and lines modeled dynamically:

- $\quad$ hybrid model 1: Line 3-4 (designated with the red color in Fig. 11)

- $\quad$ hybrid model 2: Buses 3 and 4, Lines 2-3, 3-18, 4-5 and 4-14 (designated with the blue color)

- $\quad$ hybrid model 3: Buses 2, 5, 14, 18 and 30, Lines 1-2, 2-25, 2-30, 5-6, 5-8, 13-14, 14-15, 17 18 and Generator connected to bus 30 (designated with the green color)

The number of state variables in different system models and distributed by system elements are summarized in Table 5. The overall number of state variables in the dynamic model is roughly three times higher in comparison to the steady-state model and two-times higher in comparison to hybrid model 3. 


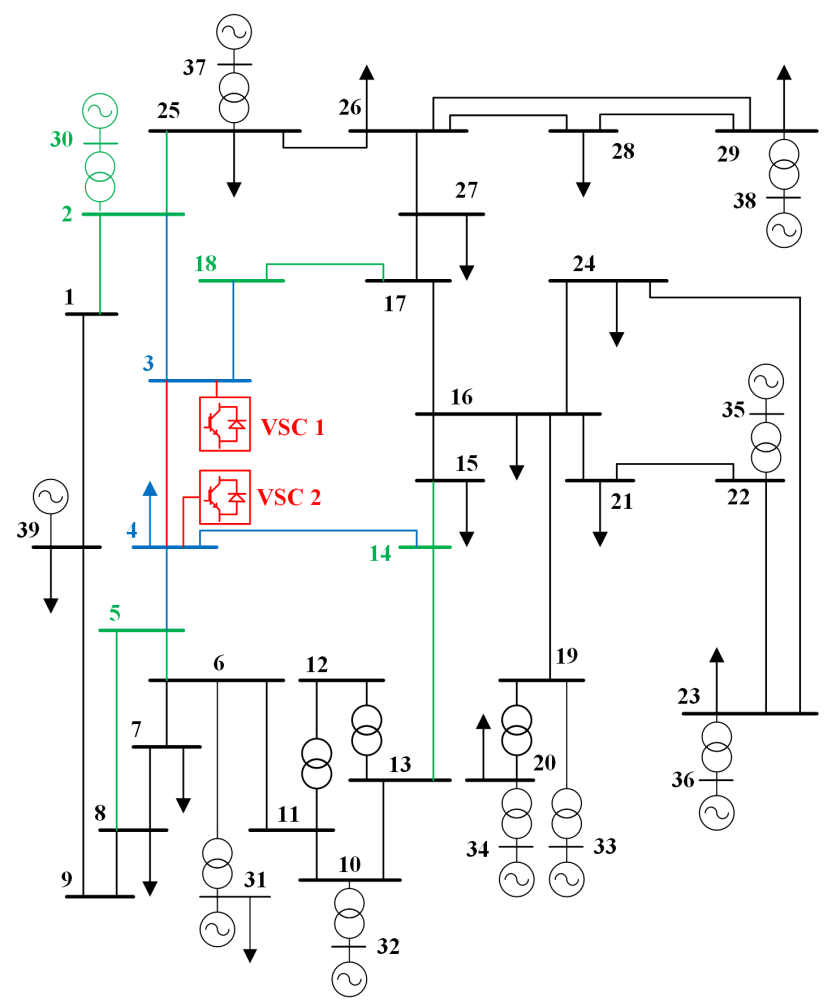

Fig. 11. IEEE 39 bus test system with two VSC stations

Table 5 Number of state variables in different IEEE 39 models with embedded MI-HVDC system

\begin{tabular}{|c|c|c|c|c|c|}
\hline $\begin{array}{c}\text { Model } \\
\text { elements }\end{array}$ & $\begin{array}{c}\text { Dynamic } \\
\text { model }\end{array}$ & $\begin{array}{c}\text { Steady-state } \\
\text { model }\end{array}$ & $\begin{array}{c}\text { Hybrid } \\
\text { model 1 }\end{array}$ & $\begin{array}{c}\text { Hybrid } \\
\text { model 2 }\end{array}$ & $\begin{array}{c}\text { Hybrid } \\
\text { model 3 }\end{array}$ \\
\hline Converters & 40 & 40 & 40 & 40 & 40 \\
\hline Ac grid & 168 & 0 & 2 & 14 & 40 \\
\hline Generators & 90 & 72 & 72 & 72 & 74 \\
\hline Overall & $\mathbf{2 9 8}$ & $\mathbf{1 1 2}$ & $\mathbf{1 1 4}$ & $\mathbf{1 2 6}$ & $\mathbf{1 5 4}$ \\
\hline
\end{tabular}

In Figs. 12a and 12b total sum errors of different models are considered, with respect to the length of line 3-4 (line interconnecting two VSC stations) for fast $\left(k_{p, P L L}=50 \mathrm{rad} / \mathrm{s}, k_{i, P L L}=\right.$ $833 \mathrm{rad} / \mathrm{s}^{2}$, Fig. 12a) and slow PLL $\left(k_{p, P L L}=20 \mathrm{rad} / \mathrm{s}, k_{i, P L L}=133 \mathrm{rad} / \mathrm{s}^{2}\right.$, Fig. 12b). The length of the line is increased two, three and four times. Since the lines in the IEEE 39 bus system are relatively short, dynamic modeling of the line, by using one PI section, is also sufficient for the lines that are up to four times longer. However, it should be noted that changing line lengths in larger grids also influences the system operating point. 
It can be observed from Fig. 12a that for longer interconnecting lines, the total sum error of different system models becomes more dispersed. Therefore, it can be concluded that more accurate modeling (with more dynamically modeled buses and lines) is more effective for a longer interconnecting line between converters. Total sum errors of different models for slow PLL speed response also become more dispersed with longer interconnecting lines, but to a much smaller extent, as can be noticed in Fig. 12b. Hence, the effectiveness of accurate ac grid modeling with a longer interconnecting line is not as pronounced as is the case for a fast PLL speed response.
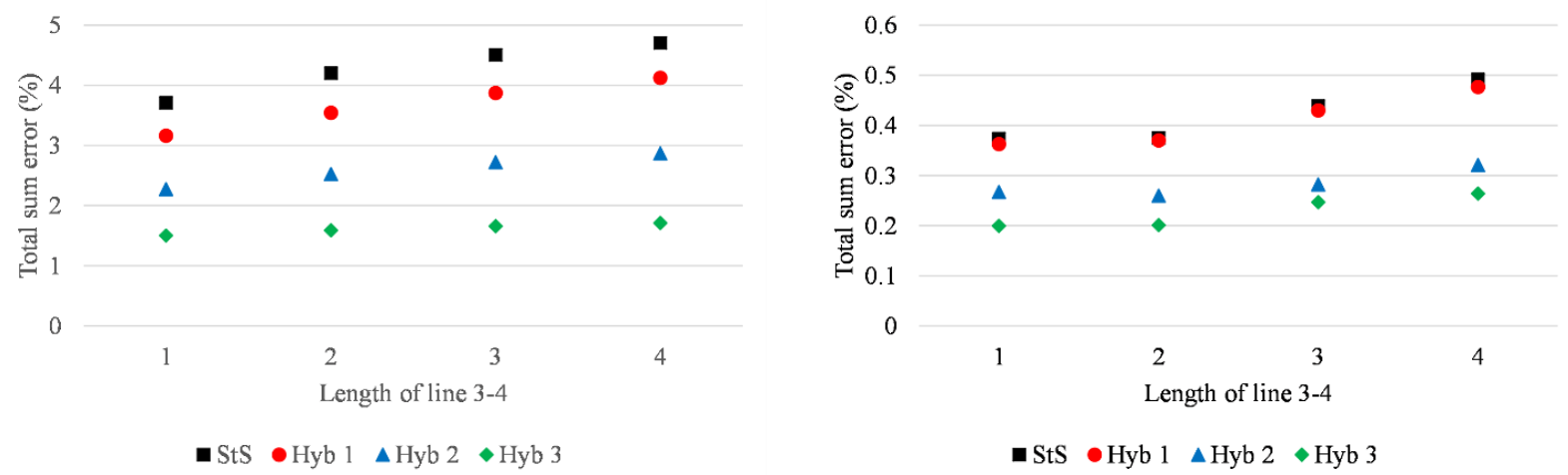

Fig. 12. Total sum error for different system models and lengths of interconnecting line 3-4: a) fast PLL; b) slow PLL

In Figs. 13a and 13b, in contrast to Figs. 12a and 12b, apart from interconnecting line 3-4, lengths of converters' neighboring lines (Lines 2-3, 3-18, 4-5, 4-14) are also increased two, three and four times. This has been carried out again for fast (Fig. 13a) and slow PLL speed responses (Fig. 13b). The total sum errors of the steady-state models become significantly higher, in comparison to the previous case, when only the interconnecting line length was increased. Hybrid 1 models, in which only the interconnecting line is modeled dynamically, reduce the error comparatively to steady-state models; but, a more significant reduction is achieved when using hybrid 2 models in which all the neighboring lines are also modeled dynamically (which corresponds to the lines whose lengths are increased in this study case). Finally, hybrid 3 models, in which additional lines are modeled dynamically, reduce the error further; but not that notably in 
comparison to the hybrid 2 model error reduction. A clear grouping of the steady-state and hybrid model 1 total sum errors, as well as hybrid model 2 and hybrid model 3 total sum errors can be observed. The total sum errors of hybrid models 2 and 3 even start to decrease with longer line lengths and fast PLL (Fig. 13a), which is not the case for a slow PLL speed response (Fig. 13b).
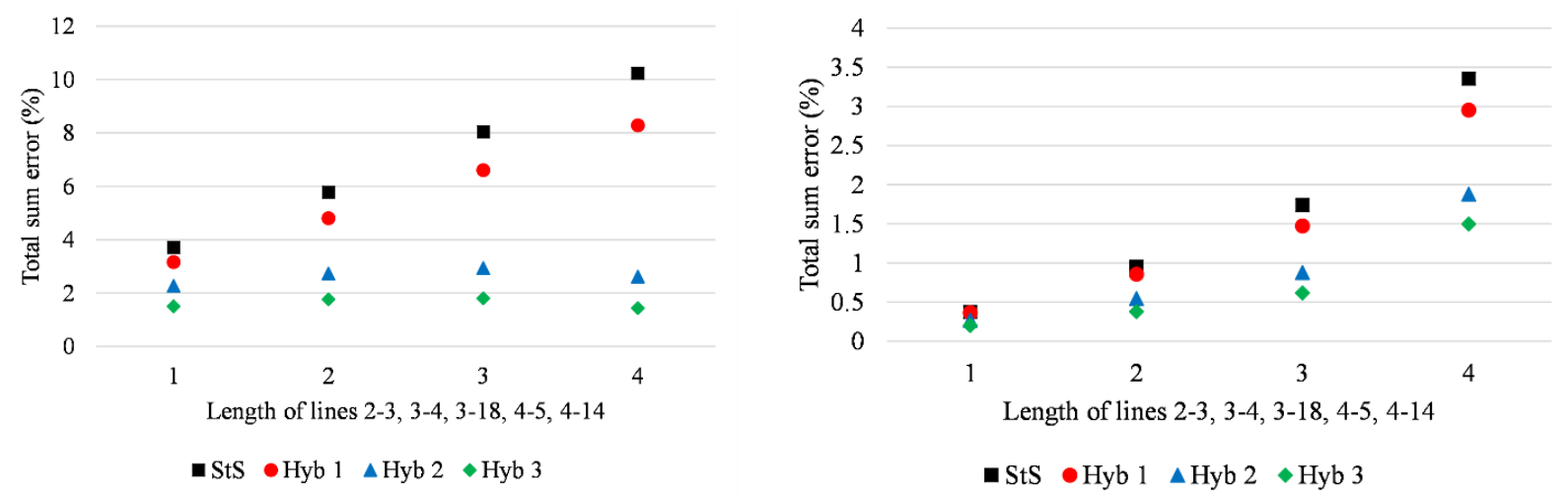

Fig. 13. Total sum error for different system models and lengths of VSC surrounding lines: a) fast PLL; b) slow PLL

\section{Conclusion}

The importance of appropriate ac grid modeling has been demonstrated in the case of a generic MI-HVDC system with two VSC converters. Instability caused by high converter's ac voltage droop coefficients remains undetected if the interconnecting ac line is not modeled dynamically.

The application of the comparison of different ac network models in the case of a larger ac system with embedded VSC HVDC converters shows that with lower SCR at the converter's point of common coupling the accuracy of the steady-state ac network model decreases. Furthermore, it has been demonstrated that among investigated converter parameters, the PLL speed control has the most significant impact on the accuracy of the system models with embedded VSC HVDC station. Therefore, in the case of fast PLL control and/or low SCR at the PCC, a larger portion of an ac grid needs to be modeled dynamically. Lastly, lengths of the converters' interconnecting and neighboring ac lines are investigated, indicating the extent of an ac grid in the vicinity of a VSC 
station which needs to be modeled dynamically. Obtained results confirm that in the case of longer ac lines around a VSC station - if those lines are modeled dynamically - a significant reduction in total sum error can be achieved. This is especially emphasized with faster PLL speed control.

\section{Acknowledgements}

The work of Jef Beerten is funded by the Research Foundation - Flanders (FWO).

\section{REFERENCES}

[1] P. Kundur, Power System Stability and Control, New York: McGraw-Hill, 1993.

[2] C. Karawita and U. D. Annakkage, "Multi-Infeed HVDC Interaction Studies Using Small-Signal Stability Assessment," IEEE Transactions on Power Delivery, vol. 24, no. 2, pp. 910-918, 2009.

[3] M. Amin, J. A. Suul, S. D'Arco, E. Tedeschi and M. Molinas, "Impact of state-space modelling fidelity on the small-signal dynamics of VSC-HVDC systems," in Proceedings of the 11th IET International Conference on $A C$ and DC Power Transmission, Birmingham, 2015.

[4] L. Zhang, L. Harnefors and H.-P. Nee, "Modeling and Control of VSC-HVDC Links Connected to Island Systems," IEEE Transactions on Power Systems, vol. 26, no. 2, pp. 783-793, 2011.

[5] G. Chunyi, L. Wei, Z. Chengyong and N. Xiaojun, "Small-signal dynamics and control parameters optimization of hybrid multiinfeed HVDC system," International Journal of Electrical Power \& Energy Systems, vol. 98, pp. 409-418, 2018.

[6] J. Z. Zhou, H. Ding, S. Fan, Y. Zhang and A. M. Gole, "Impact of Short-Circuit Ratio and Phase-Locked-Loop Parameters on the Small-Signal Behavior of a VSC-HVDC Converter," IEEE Trans. on Power Delivery, vol. 29, no. 5, pp. 2287-2296, 2014.

[7] J. Beerten, S. D'Arco and J. A. Suul, "Identification and Small-Signal Analysis of Interaction Modes in VSC MTDC Systems," IEEE Trans. on Power Delivery, vol. 31, no. 2, pp. 888-897, 2016.

[8] W. Ye, Z. Chengyong and G. Chunyi, "Comparison studyofsmall-signal stabilityofMMC-HVDC systemindifferent control modes," International Journal of Electrical Power \& Energy Systems, vol. 111, pp. 425-435, 2019.

[9] D. H. R. Suriyaarachchi, U. D. Annakkage, C. Karawita and D. A. Jacobson, "A Procedure to Study SubSynchronous Interactions in Wind Integrated Power Systems," IEEE Transactions on Power Systems, vol. 28, no. 1, pp. 377-384, 2013.

[10] D. H. R. Suriyaarachchi, U. D. Annakkage, C. Karawita, D. Kell, R. Mendis and R. Chopra, "Application of an SVC to damp sub-synchronous interaction between wind farms and series compensated transmission lines," in Proceedings of the 2012 IEEE Power and Energy Society General Meeting, San Diego, 2012.

[11] P. Hasanpor Divshali, S. H. Hosseinian and M. Abedi, "Enhancing Small Signal Stability and Reactive Powersharing Accuracy in Autonomous Microgrids by a New Decentralized Reactive Power Controller," Electric Power Components and Systems, vol. 40, no. 16, pp. 1820-1841, 2012.

[12] Y. Saad, Numerical methods for large eigenvalue problems, Society for Industrial and Applied Mathematics, 2011.

[13] C. Karawita and U. D. Annakkage, "A Hybrid Network Model for Small Signal Stability Analysis of Power Systems," IEEE Transactions on Power Systems, vol. 25, no. 1, pp. 443-451, 2010.

[14] A. Bidadfar, H.-P. Nee, L. Zhang, L. Harnefors, S. Namayantavana, M. Abedi, M. Karrari and G. B. Gharehpetian, "Power System Stability Analysis Using Feedback Control System Modeling Including HVDC Transmission Links," IEEE Transactions on Power Systems, vol. 31, no. 1, pp. 116-124, 2016. 
[15] G. Bergna Diaz, J. A. Suul and S. D'Arco, "Small-Signal State-Space Modeling of Modular Multilevel Converters for System Stability Analysis," in IEEE Energy Conversion Congress and Exposition ECCE, Montreal, 2015.

[16] G. Bergna Diaz, J. A. Suul and S. D'Arco, "Energy-Based State-Space Representation of Modulat Multilevel Converters with a Constant Equilibrium Point in Steady-State Representation," IEEE Trans. on Power Electronics, vol. 33, no. 6, pp. 4832-4851, 2018.

[17] P. W. Sauer and M. A. Pai, Power System Dynamics and Stability, Prentice Hall, 1998.

[18] IEEE, "IEEE Recommended Practice for Excitation System Models for Power System Stability Studies," 1992. [Online]. Available: https://ieeexplore.ieee.org/document/182869. [Accessed 6 May 2019].

[19] Illinois Center for a Smarter Electric Grid (ICSEG), "IEEE 39-Bus System," [Online]. Available: https://icseg.iti.illinois.edu/ieee-39-bus-system/. [Accessed 6 May 2019]. 